\title{
Força e coordenação motora da musculatura do assoalho pélvico e a função sexual feminina
}

\author{
Strength and muscle of motor coordination of pelvic \\ floor and female sexual function
}

\author{
Nina Morena Teixeira de Luccas Batista ${ }^{1,2}$, Amanda Nascimento de Oliveira ${ }^{2,3}$, \\ Erica Feio Carneiro Nunes ${ }^{4}$, Gustavo Fernando Sutter Latorre ${ }^{5}$ \\ 'Universidade Positivo, Curitiba (PR), Brasil. \\ ${ }^{2}$ Faculdade Inspirar, São Paulo (SP), Brasil. \\ ${ }^{3}$ Universidade de Santo Amaro, São Paulo (SP), Brasil. \\ ${ }^{4}$ Universidade do Estado do Pará, Belém (PA), Brasil. \\ ${ }^{5}$ Portal Perineo.net, Florianópolis (SC), Brasil.
}

Recebido: Dez. 27, 2016

Aceito: Jun. 29, 2017

\section{COMO CITAR ESTE ARTIGO}

Batista NMTL, Oliveira AN, Nunes EFC, Latorre GFS. Força e coordenação motora da musculatura do assoalho pélvico e a função sexual feminina. pélvico e a função sexual feminina.
Interdisciplinary Journal of Health Education. 2017 Jan-Jul;2(1):10-15. https:// dx.doi.org/10.4322/ijhe.2016.013

\section{CORRESPONDÊNCIA}

Gustavo Fernando Sutter Latorre Portal Perineo.net

Rua Silva Jardim, 307, Centro,

Florianópolis (SC), Brasil

Tel.: (48) 9992-8299

gustavo@perineo.net

\section{FONTE DE FINANCIAMENTO}

Nenhuma.

\section{CONFLITO DE INTERESSE}

Os autores declararam não

haver conflitos de interesse.

O estudo foi realizado na Faculdade Inspirar, São Paulo (SP), Brasil.

\section{RESUMO}

Introdução: A Organização Mundial de Saúde estipula que a sexualidade é um aspecto central do ser humano e a literatura aponta a grande participação da musculatura do assoalho pélvico (MAP) na função sexual. Objetivo: analisar a influência da força sobre a função sexual feminina. Método: foram avaliadas vinte e seis mulheres de 18 a 35 anos, com vida sexual ativa. As variáveis analisadas foram: força da MAP, desejo sexual, excitação, lubrificação, orgasmo, dor na relação e satisfação na vida sexual. A função sexual feminina foi avaliada pelo questionário FSFI - The Female Sexual Function Index e a força muscular por meio do perineômetro Perina e do toque bidigital, segundo a escala de Oxford modificada. Os dados foram analisados por meio do Software estatístico SPSS, versão 20. Estatística descritiva foi aplicada para as prevalências de disfunção sexual na amostra, e a associação entre esta disfunção e a força muscular da MAP foi investigada pelo Coeficiente de Spearman. Resultados: dentre as mulheres que apresentavam algum tipo de disfunção sexual, nenhuma possuia força da MAP igual ou superior ao grau 4 na escala de Oxford, sugerindo relação entre disfunção sexual e músculo fraco. Apesar de alguns dados tenderam à associação, não foi encontrada significância estatística. Conc/usão: os achados do presente estudo permitem concluir que parece haver correlação entre força muscular do assoalho pélvico e função sexual feminina na amostra estudada, sendo necessários estudos maiores.

PALAVRAS-Chave: Assoalho pélvico. Força muscular. Função sexual feminina. Fisioterapia pélvica.

\begin{abstract}
Background: The World Health Organization states sexuality is a central aspect of health itself. The literature points the importance of pelvic floor muscle function on female sexual function, but the subject still almost unknown. Aims: To analyze the influence of strength and coordination of pelvic floor muscles on female sexual function. Method: Twenty-six women 18 to 35 years old, sexually active, were assessed. Variables were pelvic floor muscle strength, evaluated by the Oxford scale and perineometer, and sexual function, evaluated by the FSFI on the domains sexual desire, arousal, lubrication, orgasm, pain and satisfaction. Data were analyzed descriptive statistics were applied to the prevalence of sexual dysfunction, and association between this and pelvic floor muscle strength was investigated by the coefficient Spearman. Results: Women who had some kind of sexual dysfunction had all PFM power degree 3 or less in the Oxford scale, suggesting a relationship between sexual dysfunction and weak muscle. Although some data tended to the association, no statistical significance was found. The findings of this study indicate that probably there is a correlation between muscle strength and pelvic floor female sexual function, which needs larger studies.
\end{abstract}

KEYWords: Pelvic floor. Muscle strength. Female sexual function. Pelvic physiotherapy. 


\section{Introdução}

A Organização Mundial de Saúde define que a sexualidade é um aspecto central do ser humano, vivida e expressa em pensamentos, fantasias, desejos, crenças, atitudes, valores, comportamentos, práticas, papéis e relacionamentos. É influenciada pela interação de fatores biológicos, psicológicos, sociais, econômicos, políticos, culturais, legais, históricos, religiosos e espirituais ${ }^{1}$.

Abdo et al. ${ }^{2}$ realizaram um estudo no Brasil com 1219 mulheres e revelaram que $49 \%$ tinham pelo menos uma disfunção sexual, sendo 26,7\% falta de desejo, 23,1\% dor durante a relação sexual e $21 \%$ disfunção do orgasmo ${ }^{2}$. Thiel et al. ${ }^{3}$ afirmam que a disfunção sexual feminina afeta de 30 a 50\% das mulheres americanas ${ }^{3}$. Pesquisas realizadas pelo instituto Kaplan, Centro de Estudos da Sexualidade Humana de São Paulo, relatam que a cada 100 mulheres que procuram tratamento, 70 apresentam queixas de anorgasmia ${ }^{4}$.

A literatura aponta a importância da musculatura do assoalho pélvico (MAP) na função sexual feminina, por exemplo, através do mecanismo de ereção: os músculos isquiocavernoso e bulbocavernoso se inserem no arco púbico e no clitóris de modo que, ao se contraírem na atividade sexual, puxam o clitóris para baixo, comprimindo sua drenagem venosa, facilitando a ereção $0^{5}$. Polden e Mantle ${ }^{6}$ sugerem que se a MAP em geral estiver sadia, estará mais volumosa, sendo capaz de suportar as paredes da vagina, aumentando a satisfação na relação sexual para ambos os parceiros ${ }^{6}$.

A saúde do assoalho pélvico está relacionada à consciência, à força e à resistência da MAP, de forma que essa estrutura consiga desempenhar suas funções biológicas e sexuais com êxito. Portanto, o grau de força muscular contribui sobremaneira para a satisfação sexual ${ }^{7}$.

Se a força da MAP está relacionada à melhor função sexual feminina, seria esperado que a fraqueza desta musculatura estivesse associada à disfunção sexual. No entanto, esta extrapolação a priori necessita maior investigação. Sendo assim, este estudo teve como objetivo analisar a influência da força e coordenação motora da musculatura do assoalho pélvico sobre a função sexual feminina.

\section{Métodos}

Este estudo foi aprovado pelo Comitê de Ética em Pesquisa da Faculdade Inspirar sob o número de parecer 450.048 e classificou-se como um estudo transversal Foram incluídas, por conveniência, 26 mulheres com idade entre 18 a 35 anos, com vida sexual ativa, que não apresentassem quadro de vaginismo, infecção urinária ou outro que impedisse avaliação intravaginal.

Após assinar o Termo de Consentimento Livre e Esclarecido a participante foi levada para uma sala privativa, onde respondeu ao questionário The Female Sexual Function Index $-\mathrm{FSFI}^{8}$, o índice de função sexual feminina mais utilizado mundialmente, totalmente anônimo, auto-aplicado, referente às últimas quatro semanas. Para responder às perguntas ela deveria assinalar com um " $\mathrm{X}$ " apenas uma resposta.

Na sequência, foi avaliada a força muscular do assoalho pélvico, por apenas uma pesquisadora, utilizando a Escala de Oxford ${ }^{9}$ e um manômetro vaginal, cujos valores, para fins deste artigo, significaram contração muscular máxima da MAP. Iniciando-se pela Escala de Oxford Modificada, estando a participante em posição litotômica, foram solicitadas três contrações da MAP com força máxima, como se estivesse retendo a urina, fezes e flatos, mantendo por dois segundos e com intervalo de 4 segundos entre cada contração. $\mathrm{O}$ maior valor foi registrado, conforme a Escala. Em seguida o manômetro Perina/QUARK ${ }^{\circledR}$ também foi utilizado para avaliar a força muscular, estando a participante na posição descrita anteriormente. Após insuflar a sonda e aguardar alguns segundos para adaptação ao equipamento, a pesquisadora solicitou 
as contrações perineais do mesmo modo ao realizado para a Escala de Oxford. Foram registrados os valores máximos, em $\mathrm{cm} / \mathrm{H} 2 \mathrm{O}$, das contrações efetuadas.

Ao final da avaliação as voluntárias receberam um folder elaborado pela pesquisadora, contendo informações sobre o que é assoalho pélvico, a importância dele na função sexual feminina, e como fortalecê-lo.

Os dados foram armazenados em tabelas em transferidos para o Software estatístico SPSS, versão 20. Foram consideradas portadoras de disfunção sexual feminina as voluntárias cujos escores totais do FSFI estiveram iguais ou abaixo de 26,55 , bem como dos escores de corte específicos para disfunção em cada domínio do instrumento ${ }^{10}$. Estatística descritiva foi aplicada para as prevalências de disfunção sexual na amostra, e a associação entre esta disfunção e a força muscular do assoalho pélvico foi investigada com auxílio do Coeficiente de Spearman.

\section{Resultados}

Dentre as voluntárias, 23,1\% apresentaram escores totais do FSFI compatíveis com disfunção sexual (DS) em geral. Em relação a cada domínio, 19,2\% das mulheres apresentaram escore compatível com disfunção do desejo sexual, 3,8\% compatível com disfunção de excitação, 73,1\% com disfunção de lubrificação, 46,2\% disfunção do orgasmo, 15,4\% disfunção de satisfação, 42,3\% com dor. Não houve relação entre idade e disfunção sexual.

A Tabela 1 apresenta a relação entre a força da MAP, quando avaliada pela Escala de Oxford, em função da disfunção sexual. Observa-se que, das voluntárias com escores preditivos de disfunção, nenhuma possuia força da MAP igual ou superior ao grau 4 pela escala de Oxford, apresentando, portanto, graus considerados fracos. No entanto os dados não alcançaram significância estatística $(p=0,3)$. Das mulheres sem DS, apenas 13 delas (65\%) apresentaram graus fracos (1 a 3) de força.

Quanto às médias pressóricas no manômetro, das 20 mulheres sem DS, a maioria (61\%) teve graus pressóricos superiores a $21 \mathrm{~cm} / \mathrm{H} 2 \mathrm{O}$, enquanto apenas 4 (15,3\%) apresentaram graus inferiores a $11 \mathrm{~cm} / \mathrm{H} 2 \mathrm{O}$. Para o grupo de mulheres com DS, três (50\%) apresentaram graus iguais ou inferiores a $11 \mathrm{~cm} / \mathrm{H} 2 \mathrm{O}$, e 50\% apresentaram graus iguais ou superiores a $31 \mathrm{~cm} / \mathrm{H} 2 \mathrm{O}$. Não houve, portanto, correlação entre pressão e DS (Tabela 2).

Todas as mulheres consideradas com graus fortes (4 ou 5) pela escala Oxford, apresentaram pressões superiores à $31 \mathrm{~cm} / \mathrm{H} 2 \mathrm{O}$ pelo perineômetro. No entanto, das mulheres com graus considerados fracos ( 1 a 3$), 5$ (26,3\%) foram capazes de efetuar pressões acima de $31 \mathrm{~cm} / \mathrm{H} 2 \mathrm{O}, 7$ (36,8\%) efetuaram pressões entre $21 \mathrm{e} 31 \mathrm{~cm} / \mathrm{H} 2 \mathrm{O}$, $6(31,5 \%)$ pressões entre 11 e $20 \mathrm{~cm} / \mathrm{H} 2 \mathrm{O}$, e apenas uma $(5,2 \%)$ pressões iguais ou abaixo de $10 \mathrm{~cm} / \mathrm{H} 2 \mathrm{O}$. O manômetro apresentou suficiente sensibilidade para identificar os graus fortes de contração da Escala Oxford, mas não para os mais fracos (Tabela 3).

Mulheres com disfunção no domínio dor apresentaram pressões menores $(0-20 \mathrm{~cm} / \mathrm{H} 2 \mathrm{O})$ contra mulheres sem disfunção, cujas pressões da quase totalidade foram superiores a $21 \mathrm{~cm} / \mathrm{H} 2 \mathrm{O}(\mathrm{p}=0,01)$ (Tabela 4). Houve ainda uma tendência

Tabela 1. Correlação entre disfunção sexual e força da MAP pela Escala de Oxford.

\begin{tabular}{cccc} 
& \multicolumn{2}{c}{ FORÇA PELA ESCALA DE OXFORD } & \multirow{2}{*}{ Total } \\
\cline { 2 - 4 } & Fraco & Forte & \\
\hline Normal & 13 & 7 & 6 \\
\hline DS & 6 & 0 & 6 \\
\hline
\end{tabular}

Fraco: 1 a 3; Forte: 4 e 5; DS = Disfunção sexual. 
Tabela 2. Correlação entre disfunção sexual e força da MAP pelo manômetro.

\begin{tabular}{|c|c|c|c|c|c|}
\hline & \multicolumn{4}{|c|}{ GRAUS PRESSÓRICOS } & \multirow{2}{*}{ Total } \\
\hline & 1 & 2 & 3 & 4 & \\
\hline Normal & 1 & 3 & 7 & 9 & 20 \\
\hline DS & 0 & 3 & 0 & 3 & 6 \\
\hline
\end{tabular}

Força muscular do assoalho pélvico a partir da pressão intravaginal aferida por manômetro. DS = Disfunção sexual. Graus pressóricos estratificados: $1=0$ a $10 \mathrm{~cm} / \mathrm{H} 2 \mathrm{O} ; 2=11$ a $20 \mathrm{~cm} / \mathrm{H} 2 \mathrm{O} ; 3=21$ a $30 \mathrm{~cm} / \mathrm{H} 2 \mathrm{O} ; 4=31 \mathrm{~cm} / \mathrm{H} 2 \mathrm{O}$ ou mais.

Tabela 3. Correlação entre pessão da MAP exercida no manômetro e força da MAP pela escala de Oxford.

\begin{tabular}{|c|c|c|c|c|c|}
\hline & \multicolumn{4}{|c|}{ GRAUS PRESSÓRICOS } & \multirow{2}{*}{ Total } \\
\hline & 1 & 2 & 3 & 4 & \\
\hline Fraco & 1 & 6 & 7 & 5 & 19 \\
\hline Forte & 0 & 0 & 0 & 7 & 7 \\
\hline
\end{tabular}

Fraco: 1 a 3; Forte: 4 e 5. Graus pressóricos estratificados: 1 = 0 a $10 \mathrm{~cm} / \mathrm{H} 2 \mathrm{O} ; 2=11$ a $20 \mathrm{~cm} / \mathrm{H} 2 \mathrm{O} ; 3=21$ a 30 cm/H2O; $4=31 \mathrm{~cm} / \mathrm{H} 2 \mathrm{O}$ ou mais.

Tabela 4. Correlação entre pressão da MAP exercida no manômetro e disfunção no domínio dor.

\begin{tabular}{cccccc} 
& \multicolumn{3}{c}{ GRAUS PRESSÓRICOS } & Total \\
\cline { 2 - 5 } & $\mathbf{1}$ & $\mathbf{2}$ & $\mathbf{3}$ & $\mathbf{4}$ & \\
\hline Dor ausente & 1 & 0 & 5 & 9 & 15 \\
\hline Dor presente & 0 & 6 & 2 & 3 & 11 \\
\hline
\end{tabular}

Graus pressóricos estratificados: 1 = 0 a $10 \mathrm{~cm} / \mathrm{H} 2 \mathrm{O} ; 2=11$ a $20 \mathrm{~cm} / \mathrm{H} 2 \mathrm{O} ; 3=21$ a $30 \mathrm{~cm} / \mathrm{H} 2 \mathrm{O} ; 4=31 \mathrm{~cm} / \mathrm{H} 2 \mathrm{O}$ ou mais.

na associação entre a disfunção no domínio excitação e graus pressóricos menores $(<20 \mathrm{~cm} / \mathrm{H} 2 \mathrm{O})$, no entanto esta associação não alcançou significância estatística, considerando que apenas uma das 26 mulheres esteve afetada neste domínio. Para os outros domínios não foi verificada associação entre os graus de pressão desenvolvidos e a disfunção do respectivo domínio.

\section{Discussão}

No presente estudo as mulheres que obtiveram escore compatível com disfunção sexual pelo FSFI não apresentaram grau igual ou superior a 4 na escala de Oxford modificada, sugerindo que mulheres com menor força tem mais risco de DS, o que está de acordo com o estudo de Martinez et al. ${ }^{11}$, ao avaliarem função sexual e força muscular do assoalho pélvico de 40 mulheres, utilizando o FSFI, escala de Ortiz e perineometria. O estudo mostrou que mulheres com músculos fortes pontuaram mais nos domínios de desejo, excitação, orgasmo e escore geral do questionário. Também houve correlação entre a pressão muscular do assoalho pélvico e satisfação sexual, então os autores concluíram que mulheres com MAP mais forte têm melhor função sexual ${ }^{11}$.

Uma pesquisa realizada em 2010 encontrou correlação entre orgasmo e excitação com uma melhor função da $\mathrm{MAP}^{12}$. Moura e Costa ${ }^{4}$ constataram que mulheres que alcançavam o orgasmo possuíam grau de força muscular do assoalho pélvico significativamente maior que aquelas com anorgasmia. Estes autores afirmam que 
exercícios de fortalecimento da MAP melhoram diretamente o desempenho sexual da mulher, numa intensidade variável de acordo com cada caso ${ }^{4}$.

A fisioterapia dispõe de várias técnicas de fortalecimento dessa musculatura, porém, para que ocorra o treinamento específico da MAP é necessário garantir sua correta contração. Seria pouco eficaz a realização de qualquer série de exercícios onde o movimento realizado não estivesse correto $^{13}$, daí a importância do treino de coordenação motora.

Apesar de haver uma tendência à associação, os resultados do presente estudo não mostram correlação estatisticamente significativa entre força muscular e função sexual. Esta situação poderia ser explicada pelo reduzido número de voluntárias, no entanto esta interpretação necessita cautela, pois dados similares foram descritos recentemente por Fashokun et al. ${ }^{14}$, com 505 mulheres. Os autores revelaram que os indicadores de atividade e função sexual não são distintos entre mulheres com e sem disfunção do assoalho pélvico ${ }^{14}$.

Baytur et al. ${ }^{15}$ também não encontraram correlação significativa entre força da MAP e função sexual. Eles avaliaram o tipo de parto e a força dos músculos do assoalho pélvico na função sexual de 32 mulheres que realizaram parto vaginal, 21 mulheres que se submeteram à cesariana e 15 nulíparas como controle. Os autores constataram que a força muscular do assoalho pélvico foi significativamente menor no grupo de parto normal em comparação com o grupo da cesariana e o grupo nulíparas, porém não houve diferença entre os grupos em relação à função sexual e também não houve correlação entre a função sexual e força muscular pélvica. Então concluíram que a força muscular do assoalho pélvico e o tipo de parto não afetou a função sexual em nenhuma das participantes do estudo ${ }^{15}$.

Apesar de não haver clareza na relação da força muscular do assoalho pélvico com a função sexual feminina, é importante ressaltar que não é este o único parâmetro importante desta função, nem o único capaz de ser influenciado por fisioterapia. Mendonça e Amaral ${ }^{16}$ destacam em sua revisão o importante papel da fisioterapia no incentivo ao diálogo entre o casal, carícias, jogos sexuais, fantasias e alívio da dor muscular, além do fortalecimento, mostrando que uma boa função sexual não é feita somente de um músculo forte, mas de toda uma gama de variáveis inter-relacionadas ${ }^{16}$.

Por este motivo, a sexualidade não pode ser tratada como um aspecto isolado, pois é um processo amplo e complexo que não se limita aos órgãos sexuais ou ao ato sexual ${ }^{17}$. A informação sexual pode ser influenciada por diversos fatores biológicos como: fadiga, depressão, medicações, atividade reduzida dos hormônios sexuais. Ou ainda por fatores psicológicos tais como: distrações da vida diária, receio de gravidez não desejada, doenças sexualmente transmissíveis, infertilidade comprovada, experiências sexuais negativas, inexperiência ou sentimentos de vergonha ${ }^{18}$.

De acordo com o modelo linear, tradicional, de resposta sexual, proposto na década de 1970 por Masters, Johnson e Kaplan, a primeira fase seria a do desejo, seguida pela excitação, orgasmo e resolução. Porém, modernamente, no modelo circular, proposto por Basson e colaboradores, a resposta sexual feminina consiste em fases sobrepostas de ordem variável. Ou seja, o desejo sexual pode não estar presente inicialmente, a satisfação sexual pode ocorrer sem orgasmos, estes podem ser experimentados antes da excitação máxima e mais orgasmos podem ocorrer no pico de excitação ${ }^{18}$. Já a disfunção sexual feminina é definida como qualquer desarranjo relacionado ao desejo sexual, excitabilidade, orgasmo e/ ou dor sexual ${ }^{16}$, que cause sofrimento pessoal ${ }^{18}$.

A musculatura do assoalho pélvico, localizada entre o púbis e o cóccix, e formada por músculos estriados esqueléticos ${ }^{19}$, exerce diversas funções importantes no organismo feminino como suporte aos órgãos pélvicos, manutenção da pressão responsável pelo fechamento de esfincteres uretral e anal, e função sexual ${ }^{20}$. São os músculos perivaginais e perineais do terço externo da vagina que contraem-se reflexa e ritmicamente para compor a plataforma orgásmica ${ }^{21}$.

Por fim, podemos entender que a MAP, apesar de representar grande participação na função sexual feminina, não é a única responsável pelo ciclo e, por este motivo, 
mulheres que apresentam disfunções do assoalho pélvico, como fraqueza muscular, prolapso, incontinência urinária, poderiam ter sua função sexual compensada por outros fatores. Sendo assim, uma função sexual prejudicada não implicaria em assoalho pélvico prejudicado e vice-versa.

\section{Conclusão}

Não houve correlação direta entre força muscular do assoalho pélvico e função sexual feminina na amostra estudada, embora a tendência entre graus maiores de força e maior função tenha sido observada. Apesar de alguns dados tenderam à associação, não foi encontrada significância estatística. Sugere-se a realização de novos estudos, envolvendo um número maior de participantes e controlando outras variáveis da função sexual feminina.

\section{Referências}

1. World Health Organization (WHO). Defining sexual health [Internet]. 2013 [citado em 2013 Ago 23]. Disponível em: http://www.who.int/reproductivehealth/topics/sexual health/sh definitions/en/index.html.

2. Abdo CHN, Oliveira WM Jr, Moreira ED Jr, Fittipaldi JAS. Prevalence of sexual dysfunctions and correlated conditions in a sample of Brazilian women-results of the Brazilian study on sexual behavior (BSSB). Int J Impot Res. 2004;16(2):160-6. http://dx.doi.org/10.1038/sj.ijir.3901198. PMid:14961047.

3. Thiel R, Thiel M, Palma P. Urologia feminina e medicina sexual: o que os médicos precisam saber. Prática Hospitalar. 2008;10(56):37-9.

4. Moura RVA, Costa TPB. Avaliação do grau de força do assoalho pélvico em mulheres que apresentam anorgasmia secundária [monografia]. Belém: UNAMA; 2006 [acesso em 2013 Abr 18]. Disponível em: http://www.unama.br/novoportal/ensino/ graduacao/cursos/fisioterapia/attachments/article/136/avaliacao-do-grau-de-forca-do-assoalho-pelvico.pdf

5. Silva APS, Silva JS. A importância dos músculos do assoalho pélvico feminino, sob uma visão anatômica. Fisioter Bras. 2003;4(3):205-11.

6. Polden M, Mantle J. Fisioterapia em ginecologia e obstetrícia. $2^{\mathrm{a}}$ ed. São Paulo (SP): Livraria Santos Editora; 2000.

7. Ribeiro ATA, Ankier C. A relação do grau de força muscular do assoalho pélvico com a satisfação sexual feminina. Revista Latinoamericana de Medicina Sexual - ReLAMS. 2012;1(1):14-22.

8. Rosen R, Brown C, Heiman J, et al. The Female Sexual Function Index (FSFI): a multidimensional self-report instrument for the assessment of female sexual function. J Sex Marital Ther. 2000;26(2):191-208. http://dx.doi.org/10.1080/009262300278597. PMid:10782451.

9. Gouveia PF, Ambrogini CC, Haiddar MA, Silva I. Métodos de avaliação do assoalho pélvico. RBM. 2012;70(6):232-8.

10. Wiegel M, Meston C, Rosen R. The Female Sexual Function Index (FSFI): cross-validation and development of clinical cutoff scores. J Sex Marital Ther. 2005;31(1):1-20. http://dx.doi.org/10.1080/00926230590475206. PMid:15841702.

11. Martinez CS, Ferreira FV, Castro AA, Gomide LB. Women with greater pelvic floor muscle strength have better sexual function. Acta Obstet Gynecol Scand. 2014;93(5):497-502. http://dx.doi.org/10.1111/aogs.12379. PMid:24628380.

12. Lowenstein L, Gruenwald I, Gartman I, Vardi Y. Can stronger pelvic muscle floor improve sexual function? Int Urogynecol J. 2010;21(5):553-6. http://dx.doi.org/10.1007/s00192-009-1077-5. PMid:20087572.

13. Costa TF, Resende APM, Seleme MR, Stupp L, Castro RA, Berghmans B, Sartori MGF. Ginástica hipopressiva como recurso proprioceptivo para os músculos do assoalho pélvico de mulheres incontinentes. Fisioter Bras. 2011;12(5):365-9.

14. Fashokun TBO, Harvie HS, Schimpf MO, et al. Sexual activity and function in women with and without pelvic floor disorders. Int Urogynecol J. 2013;24(1):91-7. http://dx.doi.org/10.1007/s00192-012-1848-2. PMid:22744621.

15. Baytur YB, Deveci A, Uyar Y, Ozcakir HT, Kizilkaya S, Caglar H. Mode of delivery and pelvic floor muscle strength and sexual function after childbirth. Int J Gynaecol Obstet. 2005;88(3):276-80. http://dx.doi.org/10.1016/j.ijgo.2004.12.019. PMid:15733881.

16. Mendonça CR, Amaral WN. Tratamento fisioterapêutico das disfunções sexuais femininas - Revisão de Literatura. Femina. 2011;39(3):139-42.

17. Medeiros MW, Braz MM, Brongholi K. Efeitos da fisioterapia no aprimoramento da vida sexual feminina. Fisioter bras. 2004;5(3):188-93.

18. Abdo CHN. Considerações a respeito do ciclo de resposta sexual da mulher: uma nova proposta de entendimento. Diagn Tratamento. 2010;15(2):88-90.

19. Henscher U. Fisioterapia em ginecologia. São Paulo: Editora Santos; 2007. Cap. 5; p. 83-125.

20. Grosse D, Sengler J. Reeducação perineal. Barueri: Manole; 2002.

21. Antonioli RS, Simões D. Abordagem fisioterapêutica nas disfunções sexuais femininas. Rev Neurocienc. 2010;18(2):267-74.

\section{Contribuição dos autores}

Todos os autores participaram do planejamento e execução do trabalho. 